\title{
Spatial Big Data Analytics of Influenza Epidemic in Vellore, India
}

\author{
Daphne Lopez, M. Gunasekaran, \\ B. Senthil Murugan \\ School of Information Technology \\ and Engineering \\ VIT University \\ Vellore, Tamil Nadu, India \\ daphnelopez@vit.ac.in
}

\author{
Harpreet Kaur \\ Indian Council of Medical Research \\ Government of India \\ New Delhi, India \\ kaurh@icmr.org.in
}

\author{
Kaja M. Abbas \\ Department of Population Health \\ Sciences \\ Virginia Tech \\ Blacksburg, USA \\ kaja.abbas@vt.edu
}

\begin{abstract}
The study objective is to develop a big spatial data model to predict the epidemiological impact of influenza in Vellore, India. Large repositories of geospatial and health data provide vital statistics on surveillance and epidemiological metrics, and valuable insight into the spatiotemporal determinants of disease and health. The integration of these big data sources and analytics to assess risk factors and geospatial vulnerability can assist to develop effective prevention and control strategies for influenza epidemics and optimize allocation of limited public health resources. We used the spatial epidemiology data of the HIN1 epidemic collected at the National Informatics Center during 2009-2010 in Vellore. We developed an ecological niche model based on geographically weighted regression for predicting influenza epidemics in Vellore, India during 2013-2014. Data on rainfall, temperature, wind speed, humidity and population are included in the geographically weighted regression analysis. We inferred positive correlations for H1N1 influenza prevalence with rainfall and wind speed, and negative correlations for H1N1 influenza prevalence with temperature and humidity. We evaluated the results of the geographically weighted regression model in predicting the spatial distribution of the influenza epidemic during 2013-2014.
\end{abstract}

Keywords-disease forecasting; ecological niche model; epidemiology; geographically weighted regression; H1N1 influenza

\section{INTRODUCTION}

The continuing challenge in global public health surveillance is to determine the risk posed by the infectious disease outbreaks with improved understanding of their natural geographic range. The size of the spatial epidemiology data grows large and utilization of useful intelligence in these data has become a priority. They share similar big data characteristics of volume, velocity, variety, value and veracity [1]. The spatial epidemiology data constitutes a keystone of big data and health analytics challenges in digital epidemiology [2]. This study analyzes the spatial big data challenges in infectious disease surveillance, with a focus on influenza epidemics.

\section{A. Mathematical Models of Infectious Disease Epidemics}

Mathematical models play a major role in understanding and predicting the spatiotemporal dynamics of infectious disease epidemics, and assisting in improving prevention and control policies and practices [3-6]. Bayesian networks technique is applied to model the spatio-temporal patterns of a non-contagious disease (respiratory anthrax infection) in a sample population [7-9]. Kulldroff used a spatial cluster method to group the disease cases based on location [10]. Spatial movement of individuals between locations and their contacts are monitored, grouped and visualized to control the disease spread [11].

\section{B. Spatial Analytics of Infectious Disease Epidemics}

Chi et al. summarize the application of statistical models for spatial data analysis and spatial regression modeling in population dynamics [12]. Buscema et al. applied topological weighted centroid method to predict the outbreak of Escherichia coli [13]. While the predicted results depend on the specific properties of the dataset, the parameters used to determine the utility of the predictor function are sample size, sample configuration, sample variation, distribution shape, spatial heterogeneity and spatial autocorrelation.

\section{Influenza Epidemiology in Vellore, India}

The influenza H1N1 epidemic initiated at Mexico in March 2009 , and spread globally. The influenza surveillance program in India monitored for patients with influenza symptoms. Symptoms include fever, nasal discharges, cough, headache, sore throat and respiratory problems. 10,193 cases were confirmed, with a large proportion of patients in South India, especially in Vellore. Vellore district had a high incidence of H1N1 cases, with dynamic population moving in and around Vellore. There were 433 cases reported officially; due to under-reporting, there is likely to be more than 100,000 cases that were not reported.

\section{Public Health Significance}

In this study, we have developed an ecological niche model based on geographically weighted regression for predicting influenza epidemiological impact in Vellore during 20132014, using the spatial epidemiology and ecological data of the 2009-2010 HIN1 epidemic collected at the National Informatics Center in Vellore. 


\section{METHODS}

\section{A. Spatial Autocorrelation}

Tobler's first law of geography (TFL) states "Everything is related to everything else, but near things are more related than distant things" [14-15]. Spatial autocorrelation factor is used to estimate the trueness of the Tobler's law by determining the correlation of a variable with itself over space $[14,16]$. Moran's I is a statistical measure of spatial autocorrelation [17-18]. The variations of Moran's I include Global Moran's I and Local Moran's I. Global Moran's I is used to measure the spatial autocorrelation of the entire global region and Local Moran's I is used to measure the spatial autocorrelation for each local region. Spatial autocorrelation at the local region has been used in infectious disease surveillance of dengue, HIV/AIDS and influenza to identify the hot spot locations of high disease incidence and prevalence [19-21].

\section{B. Clustering by Hot Spot - Cold Spot Analysis}

Spatial distribution of $\mathrm{H} 1 \mathrm{~N} 1$ cases are statistically calculated and clustered by Getis-Ord G statistics. It groups the spatial distribution of disease prevalence in terms of high values and low values. Clustering of high disease prevalence is referred as hotspots and clustering of low disease prevalence is referred as cold spots [22]. The hot spots and cold spots of HIN1 cases are clustered based on G score values. The computations of Getis-Ord G statistics, including the expected value are shown below.

$$
G_{i}^{*}=\frac{\sum_{j=1}^{n} W_{i j}(d) y_{j}}{\sum_{j=1}^{n} y_{j}}
$$

$G_{i}^{*}=$ G Statistic value

$W_{i j}=$ Weight matrix

$\mathrm{d}=$ Euclidean distance

$y_{j}=$ Number of infected in each location

$$
\begin{array}{ll}
E\left(G_{i}^{*}\right) & =W_{i}^{*} / n \\
W_{i}^{*} & =\sum_{j=1}^{n} W_{i j}(d)
\end{array}
$$

C. Prediction by Geographically Weighted Regression Model Traditional regression models are focused on global parameters, but the Geographically Weighted Regression (GWR) model is used to estimate the local parameters. Nonstationarity models like the geographically weighted regression model account for modeling the different observations in different locations of the study area. The geographically weighted regression model generates regression coefficients that vary over space, by estimating a separate regression coefficient for each location. Parameter $y_{i}$ denotes the prevalence of $\mathrm{H} 1 \mathrm{~N} 1$ cases in each location. It is calculated by summing up the past observations with dissimilar weights [23-24]. The model is represented as follows.
$y_{i}=\beta_{0}+\beta_{1}\left(A_{i}, B_{i}\right)+\beta_{2}\left(A_{i}, B_{i}\right) X_{1}+\ldots+\beta_{i}\left(A_{i}, B_{i}\right) X_{i k}+\varepsilon_{i k}$

$\beta_{i}\left(A_{i}, B_{i}\right)$ is a function of latitude and longitude coordinates of location $i$, and is calculated using weighted least square procedure. Specific coefficient can be calculated for each location $i$ by,

$\beta_{i}=$ Coefficient for each location $i$

$A_{i} \quad=$ Latitude

$B_{i} \quad=$ Longitude

$\hat{\beta}(i)=\left[X^{i} W(i) X\right]^{-1} X^{\prime} W(i) \quad i=1,2, \ldots, n$

Spatial observations are weighted based on the Euclidean distance between the locations $i$ and $j$. Weights $W_{i j}$ are calculated using the distance function $d_{i j}$ between the particular location $i$ and other locations, as shown below.

$$
W_{i j}=\exp \left[-\left(d_{i j}^{2} / b^{2}\right)\right]
$$

$W_{i j} \quad=\quad$ Weight of the data point $j$ at location $i$

$d_{i j}=$ Distance between the locations $i$ and $j$

$\mathrm{b}=$ Bandwidth

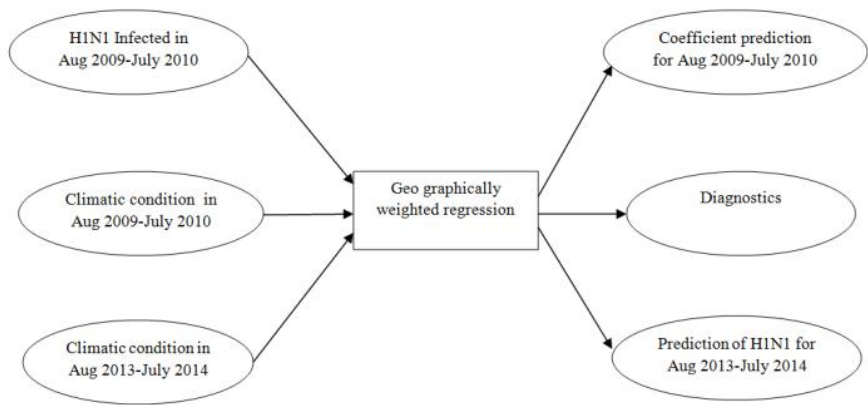

Fig. 1. Geographically Weighted Regression Model of H1N1 Influenza Epidemic. The geographically weighted regression model is calibrated using the H1N1 influenza prevalence and ecological data during 2009-2010, and used to predict the epidemiological impact of H1N1 influenza during 2013 2014

Fig. 1 illustrates the geographically weighted regression model to predict the H1N1 influenza epidemic for 2013-2014 given the H1N1 prevalence and climatic data of August 2009 to July 2010. The geographically weighted regression model finds the local regression model for each region $i$, and uses the local regression coefficient to estimate the influenza prevalence for 2013-2014. Diagnostics block are used to validate our model based on the Akaike information criteria and $R^{2}$ value. Coefficient and prediction blocks are used to generate the predicted values. The results of the geographically weighted regression model are evaluated in terms of residuals and regression coefficient. The model and simulation is implemented in ArcGIS 10.0 [25]. 


\section{RESULTS}

\section{A. Study Area}

The study area is Vellore district of Tamil Nadu state, which is located in southern India. The latitude and longitude for the Vellore district is $12.9202^{\circ} \mathrm{N}, 79.133^{\circ} \mathrm{E}$. Fig. 2 depicts the prevalence of $\mathrm{H} 1 \mathrm{~N} 1$ influenza in each administrative division of Vellore district during August 2009 to July 2010.

\section{B. Climate Conditions}

Climate condition attributes of temperature, humidity, wind speed and rainfall are collected division wise from the Vellore Agriculture Department for August 2009 to July 2010, as shown in Table I; population and H1N1 prevalence are also included in the table.

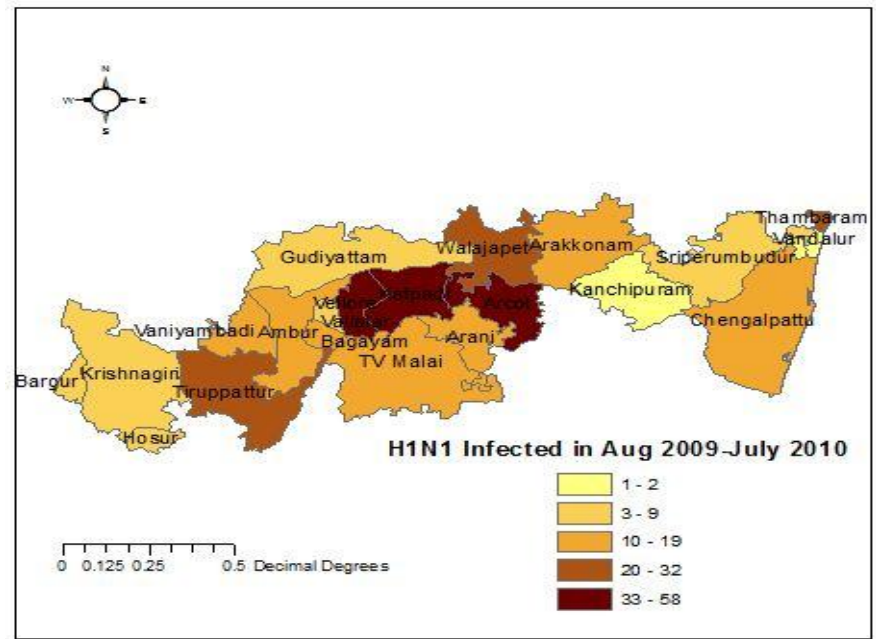

Fig. 2. H1N1 influenza prevalence during August 2009-July 2010 Prevalence of H1N1 influenza in each administrative division of Vellore district during August 2009-July 2010. Results show an outbreak in Katpadi, Arcot and Gudiyatham.

TABLE I. Climate conditions in Vellore. Climate data are derived from the Vellore Agriculture Department to include the environmental parameters that may impact the H1N1 influenza epidemic.

\begin{tabular}{|c|c|c|c|c|c|c|c|}
\hline FID & NAME_3 & Infected & Temperature & Humidity & Windspeed & Rainfall & Population \\
\hline 0 & Kelambakkam & 6 & 24.56 & 64.21 & 6 & 63 & 23453 \\
\hline 1 & Thambaram & 22 & 20.76 & 54.23 & 9 & 85 & 174787 \\
\hline 2 & Koyambedu & 1 & 35.56 & 64.98 & 4 & 56 & 12323 \\
\hline 3 & Vandalur & 1 & 35.24 & 63.45 & 4 & 59 & 13311 \\
\hline 4 & Krishnagiri & 8 & 25.02 & 66.34 & 7 & 71 & 1879809 \\
\hline 5 & Chengalpattu & 11 & 24.12 & 65.96 & 8 & 75 & 571254 \\
\hline 6 & Kanchipuram & 2 & 35.23 & 63.23 & 5 & 62 & 3998252 \\
\hline 7 & Sriperumbudur & 7 & 35.23 & 57.04 & 6 & 65 & 486063 \\
\hline 8 & Arani & 14 & 28.43 & 63.12 & 9 & 79 & 63671 \\
\hline 9 & TV Malai & 15 & 29.45 & 63.34 & 9 & 81 & 144278 \\
\hline 10 & Arakkonam & 12 & 32.65 & 64.85 & 9 & 76 & 101626 \\
\hline 11 & Arcot & 38 & 24.3 & 60.4 & 11 & 105 & 95955 \\
\hline 12 & Gudiyattam & 9 & 33.56 & 55.34 & 8 & 74 & 91558 \\
\hline 13 & Tiruppattur & 24 & 26.2 & 62.34 & 9 & 89 & 500455 \\
\hline 14 & Ambur & 16 & 27.3 & 52.76 & 10 & 83 & 114608 \\
\hline 15 & Katpadi & 43 & 22.3 & 58.57 & 12 & 112 & 387922 \\
\hline 16 & Walajapet & 32 & 25.4 & 61.34 & 11 & 95 & 32397 \\
\hline 17 & Vaniyambadi & 19 & 20.23 & 65.87 & 9 & 84 & 95061 \\
\hline 18 & Bagayam & 12 & 22.12 & 53.32 & 8 & 75 & 23145 \\
\hline 19 & Vellore & 58 & 20.3 & 65.01 & 13 & 124 & 177230 \\
\hline 20 & Vallalar & 7 & 35.1 & 64.98 & 7 & 68 & 25092 \\
\hline 21 & Hosur & 8 & 35.023 & 65.34 & 7 & 72 & 1879809 \\
\hline 22 & Bargur & 5 & 35.22 & 63.3 & 5 & 63 & 1879809 \\
\hline
\end{tabular}

\section{Correlation Analysis using Scatter plots}

Scatter plots are used to analyze the degree of correlation between temperature, humidity, wind speed, rainfall and population with H1N1 influenza prevalence, as shown in Fig.
3. We observed positive correlations between H1N1 influenza prevalence and rainfall, and between $\mathrm{H} 1 \mathrm{~N} 1$ influenza prevalence and wind speed. We observed negative correlations between $\mathrm{H} 1 \mathrm{~N} 1$ influenza prevalence and temperature, and between $\mathrm{H} 1 \mathrm{~N} 1$ influenza prevalence and humidity.

\section{Clustering by Hot Spot - Cold Spot Analysis}

Fig. 4 depicts the hot spot and cold spot locations for the H1N1 epidemic during the winter season. The hot spot is observed in Arani and the cold spot in Sriperumbudur.

\section{E. Parameter Values}

The values of the parameters used for prediction are shown in Table II.

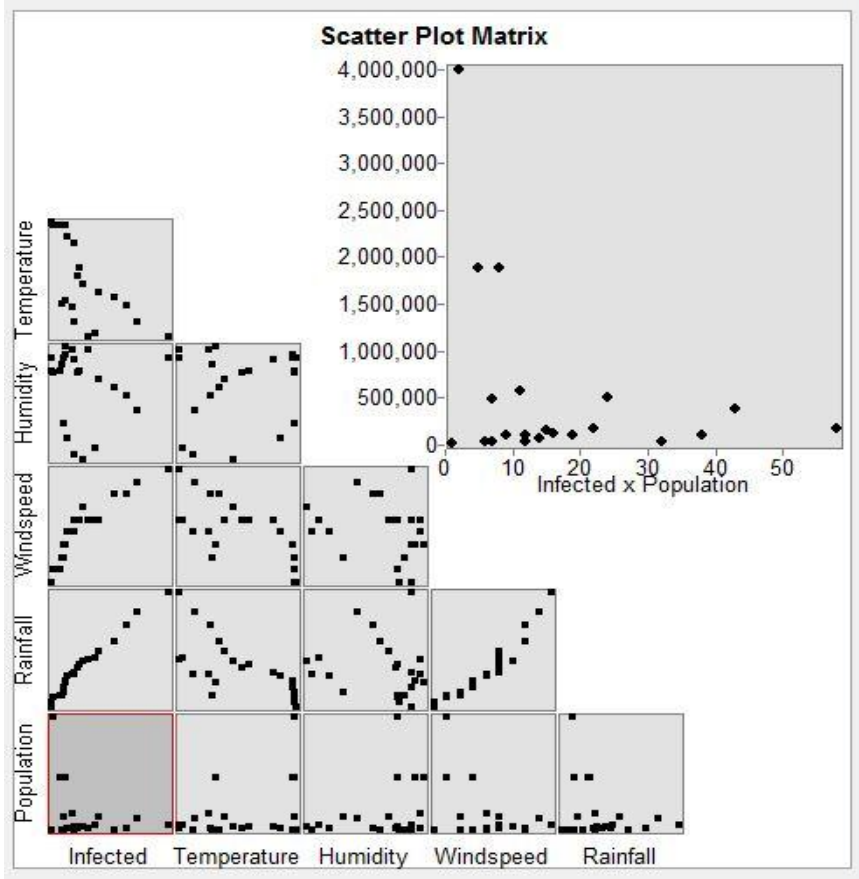

Fig. 3. Correlation analysis using scatter plots. Analysis of the scatter plots infer that rainfall and windspeed have a positive correlation, whereas temperature and humidity have a negative correlation on $\mathrm{H} 1 \mathrm{~N} 1$ influenza prevalence.

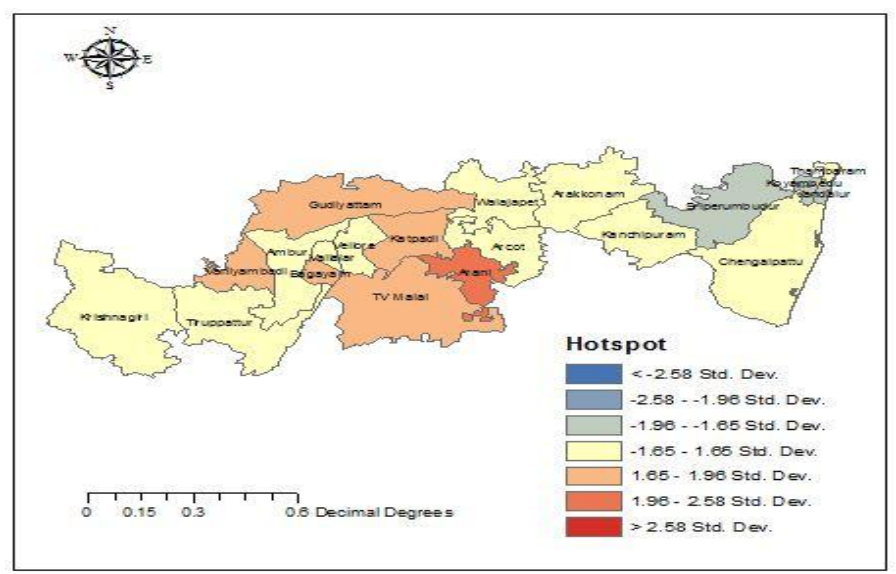

Fig. 4. H1N1 hotspot - cold spot analysis. Arani is identified as the hot spot and Sriperumbuthur is identified to be a cold spot for H1N1 influenza. 
TABLE II. Parameters' Estimation Using Ordinary Least Squares. Linear regression analysis of correlation between H1N1 influenza prevalence and rainfall, wind speed, humidity and temperature using ordinary least squares estimation method.

\begin{tabular}{|c|c|c|c|}
\hline \multirow{4}{*}{} & Variable & Co-efficient & P value \\
\cline { 2 - 4 } & Rainfall & 0.2013 & 0.0012 \\
\cline { 2 - 4 } OLS & Wind speed & 1.0232 & 0.0032 \\
\cline { 2 - 4 } & Humidity & -1.0197 & 0.0234 \\
\cline { 2 - 4 } & Temperature & -1.3423 & 0.0487 \\
\cline { 2 - 4 } & $R^{2}$ & \multicolumn{2}{|c|}{0.924875} \\
\cline { 2 - 4 } & Adjusted $R^{2}$ & \multicolumn{2}{|c|}{0.914620} \\
\cline { 2 - 4 } & AIC & \multicolumn{2}{|c|}{124.5678} \\
\hline
\end{tabular}

\section{F. Residuals}

The residuals measure the difference between the predicted and actual values using ordinary least squares. Fig. 5 shows the spatial distribution of the residuals. In order to validate the parametric values within the neighborhood, geographically weighted regression and spatial autocorrelation is applied. GWR spatial autocorrelation significance is shown in Table III and proves to have a correlation with the derived GWR model.

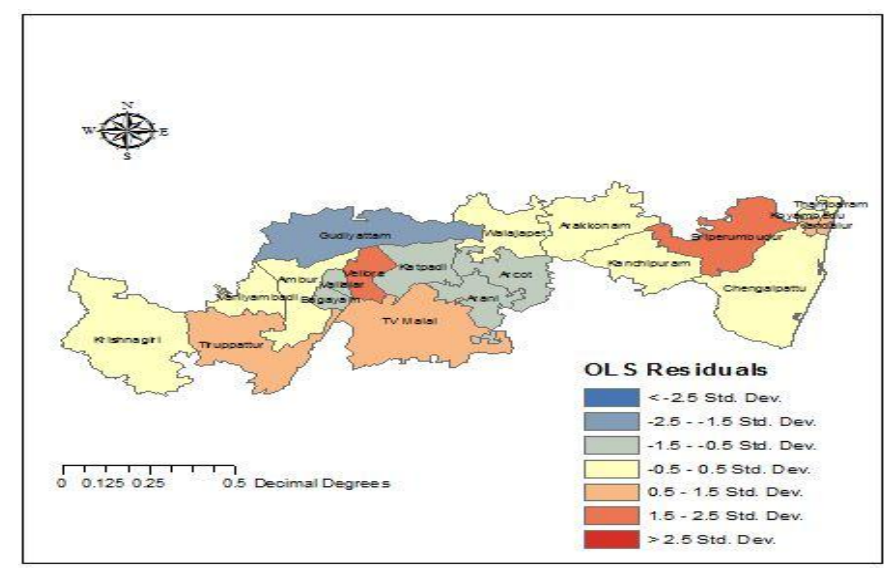

Fig. 5. Ordinary least squares residuals. The spatial distribution of the residuals is mapped.

TABLE III. Spatial autocorrelation for residuals in geographically weighted regression model. The $p$-value is statistically significant and the $z$ score is positive, thereby inferring spatial autocorrelation between the spatial locations and the feature values.

\begin{tabular}{|c|c|c|}
\hline \multirow{3}{*}{ GWR } & Moran's Index & 0.249226 \\
\cline { 2 - 3 } & Expected Index & 0.050000 \\
\cline { 2 - 3 } & Variance $\mathbf{Z}$ score & 0.009890 \\
\cline { 2 - 3 } & $\mathbf{Z}$ score & 1.003338 \\
\cline { 2 - 3 } & P value & 0.045141 \\
\hline
\end{tabular}

\section{G. Regression Coefficient Prediction}

After identifying the correlations using data from 20092010, regression coefficients are used to predict the H1N1 prevalence in each location during 2013-2014. Regression coefficients are calculated and shown in Table IV and Table $\mathrm{V}$.
TABLE IV. Coefficient prediction for August 2009-July2010. The coefficients are predicted using the data from the 2009-2010 H1N1 influenza season.

\begin{tabular}{|r|r|r|r|r|r|r|r|}
\hline FID & LocalR2 & Predicted & Intercept & C2_Rainfall & C3_Wind & C1_Hum & C1_Temp \\
\hline 0 & 0.941536 & 4.272267 & 65.96521 & 0.20191 & 1.569884 & -1.01122 & -1.2123 \\
\hline 1 & 0.941347 & 7.57038 & 65.8725 & 0.20017 & 1.57597 & -1.01176 & -1.20799 \\
\hline 2 & 0.941604 & 7.218828 & 65.97145 & 0.201849 & 1.387915 & -1.0117 & -1.21268 \\
\hline 3 & 0.941484 & 4.096468 & 65.89729 & 0.200351 & 1.474089 & -1.01174 & -1.20928 \\
\hline 4 & 0.976791 & 7.394924 & 72.69193 & 0.399978 & 1.513415 & -1.01295 & -1.55314 \\
\hline 5 & 0.94231 & 15.50616 & 66.18829 & 0.205244 & 1.390119 & -1.01159 & -1.22327 \\
\hline 6 & 0.944341 & 5.702962 & 67.2873 & 0.227909 & 1.461877 & -1.01125 & -1.27439 \\
\hline 7 & 0.942483 & 4.272507 & 66.45469 & 0.211483 & 1.55629 & -1.01147 & -1.23504 \\
\hline 8 & 0.962413 & 13.89707 & 72.5177 & 0.339014 & 1.513841 & -1.01297 & -1.53259 \\
\hline 9 & 0.968666 & 4.273444 & 73.07605 & 0.372021 & 1.570263 & -1.01258 & -1.56524 \\
\hline 10 & 0.945692 & 12.46897 & 67.96397 & 0.242234 & 1.570797 & -1.01119 & -1.30589 \\
\hline 11 & 0.954576 & 36.2268 & 70.6383 & 0.297585 & 0.62999 & -1.01189 & -1.43766 \\
\hline 12 & 0.971071 & 8.998451 & 73.36439 & 0.383539 & 1.431956 & -1.01276 & -1.57934 \\
\hline 13 & 0.975043 & 9.083651 & 72.81947 & 0.39311 & 1.583877 & -1.01264 & -1.55772 \\
\hline 14 & 0.973581 & 5.962924 & 73.01804 & 0.389487 & 1.497208 & -1.01253 & -1.56559 \\
\hline 15 & 0.967572 & 61.02714 & 73.34657 & 0.369609 & 1.451583 & -1.0131 & -1.57601 \\
\hline 16 & 0.95332 & 15.50215 & 70.38698 & 0.292211 & 1.526968 & -1.01177 & -1.42401 \\
\hline 17 & 0.974829 & 7.566075 & 72.92495 & 0.393613 & 1.426165 & -1.01264 & -1.56218 \\
\hline 18 & 0.972607 & 4.273573 & 73.07329 & 0.386307 & 1.540191 & -1.0125 & -1.56748 \\
\hline 19 & 0.970729 & 45.7655 & 73.24372 & 0.381163 & 1.26015 & -1.01263 & -1.57387 \\
\hline 20 & 0.972231 & 7.394772 & 73.14261 & 0.385677 & 1.518715 & -1.01253 & -1.57031 \\
\hline 21 & 0.97651 & 5.877111 & 72.65238 & 0.397816 & 1.494516 & -1.01289 & -1.55128 \\
\hline 22 & 0.977283 & 2.671446 & 72.63378 & 0.40186 & 1.419994 & -1.01307 & -1.55091 \\
\hline
\end{tabular}

TABLE V. Predicted values of H1N1 influenza prevalence during 2013-2014. The epidemiological impact of H1N1 influenza during 2013-2014 is predicted by the geographic weighted regression model.

\begin{tabular}{|r|r|r|r|r|r|r|r|}
\hline FID & LocalR2 & Predicted & Intercept & C2_Rainfall & C3_Wind & C1_Hum & C1_Temp \\
\hline 0 & 0.941536 & 9.796861 & 65.96521 & 0.20191 & 1.569884 & -1.01122 & -1.2123 \\
\hline 1 & 0.941347 & 11.67607 & 65.8725 & 0.20017 & 1.57597 & -1.01176 & -1.20799 \\
\hline 2 & 0.941604 & -0.92398 & 65.97145 & 0.201849 & 1.387915 & -1.0117 & -1.21268 \\
\hline 3 & 0.941484 & 1.967236 & 65.89729 & 0.200351 & 1.474089 & -1.01174 & -1.20928 \\
\hline 4 & 0.976791 & 4.567098 & 72.69193 & 0.399978 & 1.513415 & -1.01295 & -1.55314 \\
\hline 5 & 0.94231 & 19.17416 & 66.18829 & 0.205244 & 1.390119 & -1.01159 & -1.22327 \\
\hline 6 & 0.944341 & 1.003856 & 67.2873 & 0.227909 & 1.461877 & -1.01125 & -1.27439 \\
\hline 7 & 0.942483 & 5.944097 & 66.45469 & 0.211483 & 1.55629 & -1.01147 & -1.23504 \\
\hline 8 & 0.962413 & 13.78208 & 72.5177 & 0.339014 & 1.513841 & -1.01297 & -1.53259 \\
\hline 9 & 0.968666 & 16.70506 & 73.07605 & 0.372021 & 1.570263 & -1.01258 & -1.56524 \\
\hline 10 & 0.945692 & 13.87228 & 67.96397 & 0.242234 & 1.570797 & -1.01119 & -1.30589 \\
\hline 11 & 0.954576 & 3.91911 & 70.6383 & 0.297585 & 0.62999 & -1.01189 & -1.43766 \\
\hline 12 & 0.971071 & 5.358784 & 73.36439 & 0.383539 & 1.431956 & -1.01276 & -1.57934 \\
\hline 13 & 0.975043 & 15.49282 & 72.81947 & 0.39311 & 1.583877 & -1.01264 & -1.55772 \\
\hline 14 & 0.973581 & 21.45832 & 73.01804 & 0.389487 & 1.497208 & -1.01253 & -1.56559 \\
\hline 15 & 0.967572 & 25.1756 & 73.34657 & 0.369609 & 1.451583 & -1.0131 & -1.57601 \\
\hline 16 & 0.95332 & 18.11901 & 70.38698 & 0.292211 & 1.526968 & -1.01177 & -1.42401 \\
\hline 17 & 0.974829 & 21.43777 & 72.92495 & 0.393613 & 1.426165 & -1.01264 & -1.56218 \\
\hline 18 & 0.972607 & 7.055796 & 73.07329 & 0.386307 & 1.540191 & -1.0125 & -1.56748 \\
\hline 19 & 0.970729 & 29.55596 & 73.24372 & 0.381163 & 1.26015 & -1.01263 & -1.57387 \\
\hline 20 & 0.972231 & 13.92465 & 73.14261 & 0.385677 & 1.518715 & -1.01253 & -1.57031 \\
\hline 21 & 0.97651 & 4.135098 & 72.65238 & 0.397816 & 1.494516 & -1.01289 & -1.55128 \\
\hline 22 & 0.977283 & 1.119658 & 72.63378 & 0.40186 & 1.419994 & -1.01307 & -1.55091 \\
\hline
\end{tabular}


The spatial distribution of the coefficient values for rainfall, temperature, wind speed and humidity are shown in Fig. 6, Fig. 7, Fig. 8 and Fig. 9 respectively.

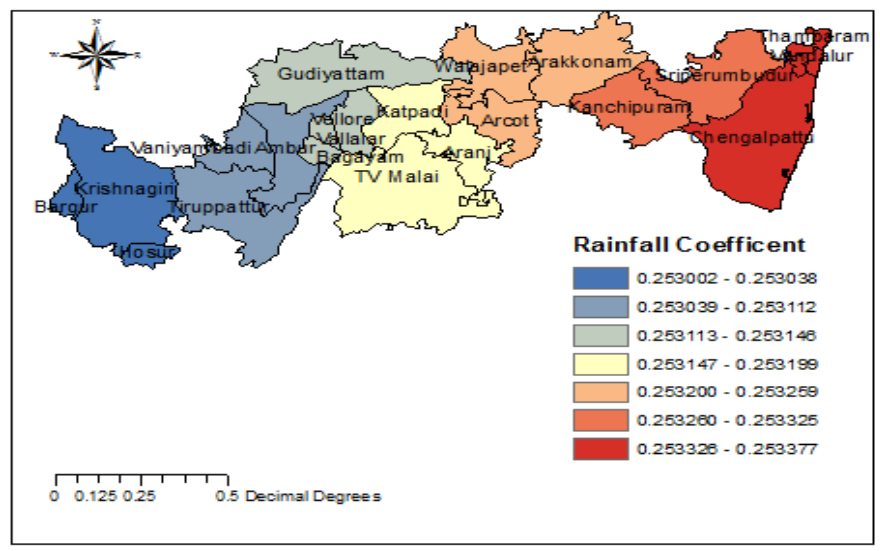

Fig. 6. Rainfall coefficient. Rainfall is positively correlated with the prevalence of H1N1 influenza. Areas around Chengalpatu has high rainfall coefficients compared to other locations, and the neighbourhood locations of Krishnagiri has lower rainfall coefficients.

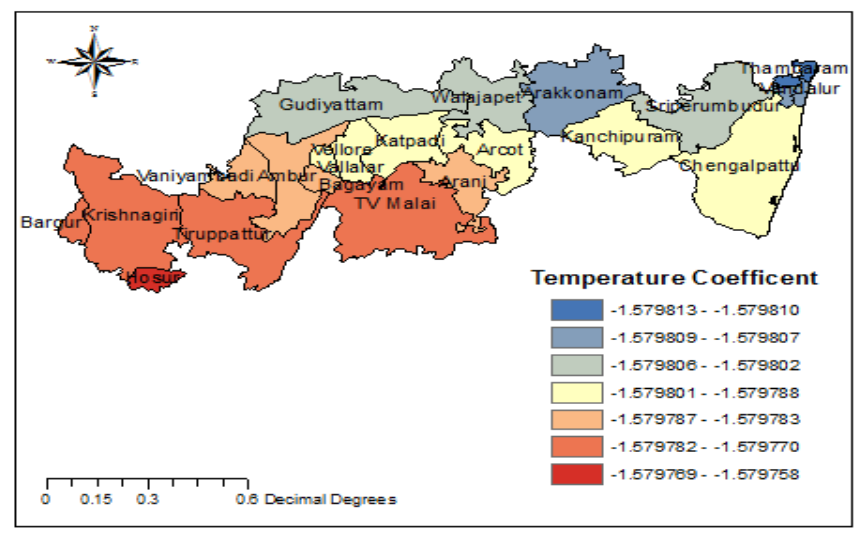

Fig. 7. Temperature coefficient. Temperature is negatively correlated with the prevalence of H1N1 influenza.TV Malai, Krishnagiri, Thirupattur has low temperature coefficients while Arakonam and Thambaram have high temperature coefficients.

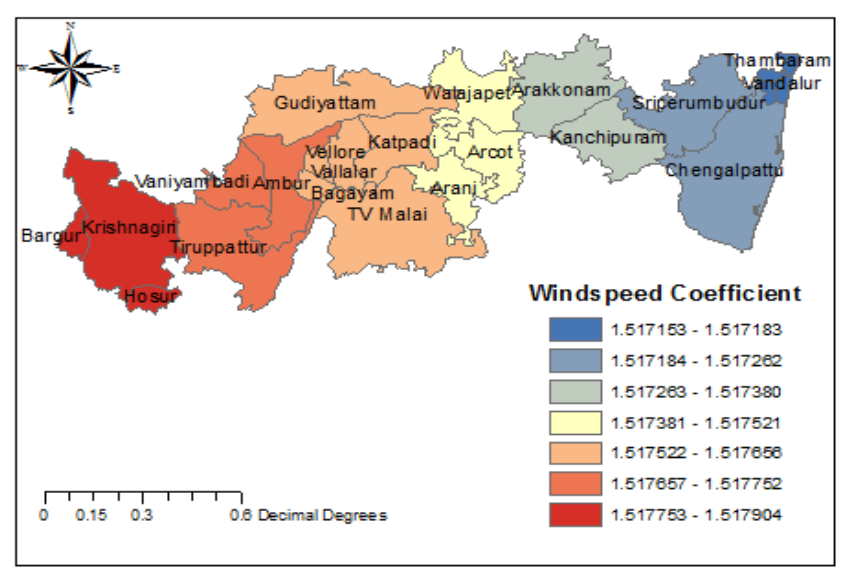

Fig. 8. Windspeed coefficient. Windspeed is positively correlated with the prevalence of H1N1 influenza. Areas around Krishnagiri have high windspeed and areas near Thambaram have low windspeed.

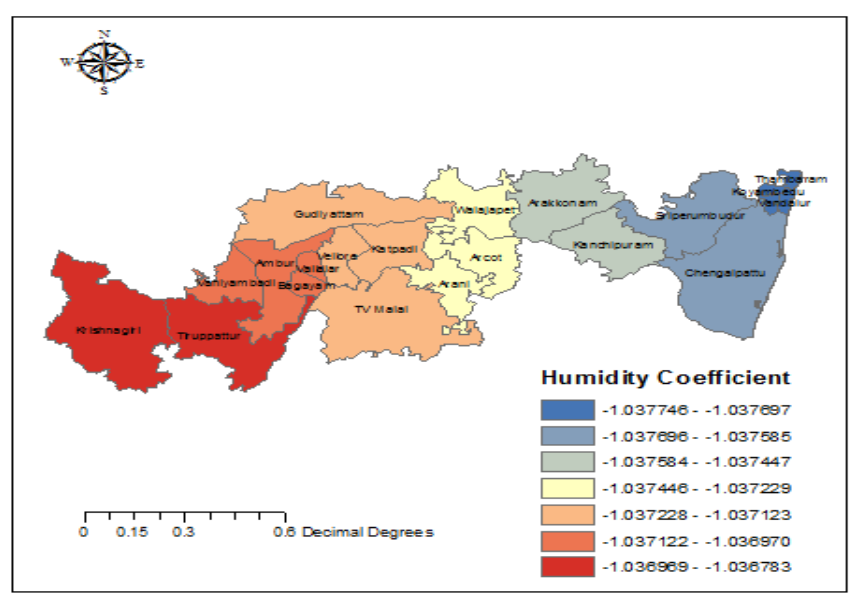

Fig. 9. Humidity coefficient. Humidity is negatively correlated with the prevalence of H1N1 influenza. Areas near Krishnagiri and Tirupattur have high humidity and Chengalpattu has low humidity.

The spatial distribution of H1N1 influenza prevalence is predicted for each location, and is shown in Fig. 10. The predictions for 2013-2014 are based on the regression coefficients estimated using the data from 2009-2010.

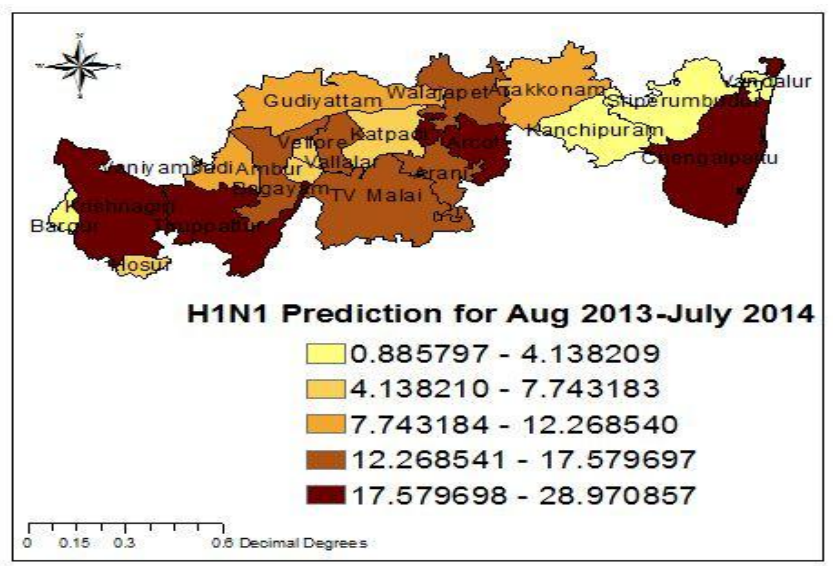

Fig. 10. Prediction of H1N1 influenza. The spatial distribution of H1N1 infuenza prevalence for 2013-2014 is predicted.

\section{DISCUSSION}

We developed an ecological niche model based on geographically weighted regression method to predict the epidemiological impact of H1N1 influenza during 2013-2014 season. We integrated the spatial epidemiology data of H1N1 influenza prevalence and environmental data from 2009-2010 season. We inferred that $\mathrm{H} 1 \mathrm{~N} 1$ influenza prevalence has positive correlations with rainfall and wind speed, and negative correlations with temperature and humidity.

\section{A. Limitations}

The ecological niche model based on geographically weighted regression is used to predict the spatial distribution of $\mathrm{H} 1 \mathrm{~N} 1$ influenza prevalence. While the environmental variables of rainfall, wind speed, temperature and humidity correlate to the risk of influenza incidence and prevalence in different regions, biological and socio-behavioral attributes of 
H1N1 influenza transmission dynamics are not incorporated in the model.

\section{B. Public Health Implications}

The ecological niche model based on geographically weighted regression is used to predict the epidemiological impact of H1N1 influenza in different regions. Thereby, high risk areas for $\mathrm{H} 1 \mathrm{~N} 1$ influenza can be prioritized for implementation of prevention interventions.

\section{Conclusion}

Epidemiological models of infectious diseases are useful to predict the epidemiological morbidity and mortality, identify vulnerable populations, assess the beneficial impact of available interventions, compare different implementation options, and improve public understanding of infectious disease dynamics [26]. We presented the ecological niche model based on geographically weighted regression to predict the incidence and prevalence of $\mathrm{H} 1 \mathrm{~N} 1$ influenza in different regions of Vellore, India, thereby assisting in prioritizing high risk areas for implementation of optimal prevention interventions.

The integration of health, climate and environmental data, supported by geographical information systems and satellite imagery, and combined with computational tools facilitate the design and development of early warning systems for influenza epidemics, and can be adapted to control and prevent epidemics of other infectious diseases.

\section{ACKNOWLEDGEMENTS}

DL and MG are supported in part by the Indian Council of Medical Research under Award Number 32/1/2010-ECD-I. KMA is supported in part by the National Institute of General Medical Sciences of the National Institutes of Health under Award Number R01GM109718. The content is solely the responsibility of the authors and does not necessarily represent the official views of the funding agencies.

\section{REFERENCES}

[1] J. Burke, Health Analytics: Gaining the Insights to Transform Health Care, 1 edition. Hoboken, New Jersey: Wiley, 2013.

[2] M. Salathé, L. Bengtsson, T. J. Bodnar, D. D. Brewer, J. S. Brownstein, C. Buckee, E. M. Campbell, C. Cattuto, S. Khandelwal, P. L. Mabry, and A. Vespignani, "Digital Epidemiology," PLoS Comput Biol, vol. 8, no. 7, p. e1002616, 2012.

[3] E. Vynnycky and R. White, An Introduction to Infectious Disease Modelling, 1st ed. Oxford University Press, USA, 2010.

[4] M. J. Keeling and P. Rohani, Modeling Infectious Diseases in Humans and Animals, 1st ed. Princeton University Press, 2007.

[5] D. U. Pfeiffer, T. P. Robinson, M. Stevenson, K. B. Stevens, D. J. Rogers, and A. C. A. Clements, Spatial Analysis in Epidemiology. Also available as: Hardback | eBook, 2008.

[6] L. A. Waller and C. A. Gotway, Applied Spatial Statistics for Public Health Data, 1 edition. Hoboken, N.J: Wiley-Interscience, 2004.

[7] X. Jiang and G. Wallstrom, "A Bayesian Network for Outbreak Detection and Prediction," in Proceedings of AAAI-06, Boston, MA, 2006.

[8] G. F. Cooper, D. H. Dash, J. D. Levander, W.-K. Wong, W. R. Hogan, and M. M. Wagner, "Bayesian Biosurveillance of Disease Outbreaks," in Proceedings of the 20th Conference on Uncertainty in Artificial Intelligence, Arlington, Virginia, United States, 2004, pp. 94-103.

[9] D. Neill, A. Moore, and G. Cooper, "A Bayesian Spatial Scan Statistic," Adv. Neural Inf. Process. Syst., vol. 18, 2005.
[10] M. Kulldorff, "A spatial scan statistic," Commun. Stat. - Theory Methods, vol. 26, no. 6, pp. 1481-1496, Jan. 1997.

[11] T. C. Germann, K. Kadau, I. M. Longini, and C. A. Macken, "Mitigation strategies for pandemic influenza in the United States," Proc. Natl. Acad. Sci. U. S. A., vol. 103, no. 15, pp. 5935-5940, Apr. 2006

[12] G. Chi and J. Zhu, "Spatial Regression Models for Demographic Analysis," Popul. Res. Policy Rev., vol. 27, no. 1, pp. 17-42, Feb. 2008.

[13] M. Buscema, E. Grossi, A. Bronstein, W. Lodwick, M. AsadiZeydabadi, R. Benzi, and F. Newman, "A New Algorithm for Identifying Possible Epidemic Sources with Application to the German Escherichia coli Outbreak," ISPRS Int. J. Geo-Inf., vol. 2, no. 1, pp. 155-200, Mar. 2013.

[14] H. J. Miller, "Tobler's First Law and Spatial Analysis," Ann. Assoc. Am. Geogr., vol. 94, no. 2, pp. 284-289, Jun. 2004.

[15] W. R. Tobler, "A Computer Movie Simulating Urban Growth in the Detroit Region," Econ. Geogr., vol. 46, pp. 234-240, Jun. 1970.

[16] S. Farber, A. Páez, and E. Volz, "Topology and Dependency Tests in Spatial and Network Autoregressive Models," Geogr. Anal., vol. 41, no. 2 , pp. 158-180, Apr. 2009.

[17] D. E. Impoinvil, T. Solomon, W. W. Schluter, A. Rayamajhi, R. P. Bichha, G. Shakya, C. Caminade, and M. Baylis, "The spatial heterogeneity between Japanese encephalitis incidence distribution and environmental variables in Nepal," PloS One, vol. 6, no. 7, p. e22192, 2011.

[18] M. M. Wall, "A close look at the spatial structure implied by the CAR and SAR models," J. Stat. Plan. Inference, vol. 121, no. 2, pp. 311324, Apr. 2004

[19] C.-H. Lin, K. L. Schiøler, M. R. Jepsen, C.-K. Ho, S.-H. Li, and F. Konradsen, "Dengue outbreaks in high-income area, Kaohsiung City, Taiwan, 2003-2009," Emerg. Infect. Dis., vol. 18, no. 10, pp. 16031611, Oct. 2012.

[20] J. Phaisarn, "Spatial Patterns Analysis and Hotspots of HIV/AIDS in Phayao Province, Thailand," Arch. Sci., vol. 65, no. 9, 2012.

[21] J. H. Stark, R. Sharma, S. Ostroff, D. A. T. Cummings, B. Ermentrout, S. Stebbins, D. S. Burke, and S. R. Wisniewski, "Local spatial and temporal processes of influenza in Pennsylvania, USA: 2003-2009," PloS One, vol. 7, no. 3, p. e34245, 2012.

[22] L. Anselin, "Local Indicators of Spatial Association-LISA," Geogr. Anal., vol. 27, no. 2, pp. 93-115, Apr. 1995.

[23] A. S. Fotheringham, C. Brunsdon, and M. Charlton, Geographically Weighted Regression: The Analysis of Spatially Varying Relationships. John Wiley \& Sons, 2003.

[24] C. Brunsdon, A. S. Fotheringham, and M. Charlton, "Some Notes on Parametric Significance Tests for Geographically Weighted Regression," J. Reg. Sci., vol. 39, no. 3, pp. 497-524, Aug. 1999.

[25] ArcGIS, Geographic Information Systems Software. ESRI, 2014.

[26] H. V. Fineberg and M. E. Wilson, "Epidemic science in real time," Science, vol. 324, no. 5930, p. 987, May 2009 